\title{
Serotonergic neurons in the caudal raphe nuclei discharge in association with activity of masticatory muscles
}

L.E. Ribeiro-do-Valle ${ }^{1}$
1Departamento de Fisiologia e Biofísica, Instituto de Ciências Biomédicas, Universidade de São Paulo, 05508-900 São Paulo, SP, Brasil

\section{Correspondence \\ L.E. Ribeiro-do-Valle \\ Departamento de Fisiologia e \\ Biofísica \\ Instituto de Ciências Biomédicas \\ Universidade de São Paulo \\ 05508-900 São Paulo, SP \\ Brasil \\ E-mail: ribeiro@bmb.icb1.usp.br \\ Research supported by FAPESP and CNPq.}

Received June 4, 1996 Accepted November 11, 1996

\begin{abstract}
There is a dense serotonergic projection from nucleus raphe pallidus and nucleus raphe obscurus to the trigeminal motor nucleus and serotonin exerts a strong facilitatory action on the trigeminal motoneurons. Some serotonergic neurons in these caudal raphe nuclei increase their discharge during feeding. The objective of the present study was to investigate the possibility that the activity of these serotonergic neurons is related to activity of masticatory muscles. Cats were implanted with microelectrodes and gross electrodes. Caudal raphe single neuron activity, electrocorticographic activity, and splenius, digastric and masseter electromyographic activities were recorded during active behaviors (feeding and grooming), during quiet waking and during sleep. Seven presumed serotonergic neurons were identified. These neurons showed a long duration action potential $(>2.0$ msec), and discharged slowly $(2-7 \mathrm{~Hz})$ and very regularly (interspike interval coefficient of variation $<0.3$ ) during quiet waking. The activity of these neurons decreased remarkably during fast wave sleep (78$100 \%)$. Six of these neurons showed tonic changes in their activity positively related to digastric and/or masseter muscles activity but not to splenius muscle activity during waking. These data are consistent with the hypothesis that serotonergic neurons in the caudal raphe nuclei play an important role in the control of jaw movements.
\end{abstract}

The trigeminal motor nucleus receives a dense serotonergic projection $(1,2)$ which originates from nucleus raphe pallidus, nucleus raphe obscurus and, to a lesser extent, from nucleus raphe dorsalis $(3,4)$. Serotonergic fibers terminate in close proximity to the cell body and proximal processes of trigeminal motoneurons $(1,5)$. Serotonin was shown to strongly facilitate trigeminal motoneurons $(6,7)$. These findings suggest an important role for the serotonergic system in
Key words

- Masticatory muscles

- Serotonergic neurons

- Caudal raphe nuclei

- Feeding behavior

- Grooming behavior the control of jaw muscles activity.

It is well known that the activity of the serotonergic neurons in the raphe nuclei is related to the level of arousal of the organism (for a review, see Ref. 8). Thus, the tonic serotonergic influence on the trigeminal motoneurons during waking may contribute to maintaining jaw position and facilitating jaw movements during feeding, grooming, and defense/aggression behaviors.

It has been shown that some serotonergic 
neurons in nucleus raphe dorsalis selectively increase their activity during feeding and grooming behaviors $(9,10)$. Many serotonergic neurons in nucleus raphe pallidus and nucleus raphe obscurus behave similarly (11). These results raise the interesting possibility that there may be additional changes in the serotonergic influence on the trigeminal motoneurons associated with the activation of these motoneurons by central mechanisms during behaviors that involve oral movements. In the present study we investigated this hypothesis.

Adult cats of either sex were used. Housing conditions of the animals and the experimental protocol were reviewed and approved by the Comissão de Fiscalização de Pesquisa com Animais (COFIPA) of São Paulo City. For single neuron recording the animals were stereotaxically implanted with a microdrive consisting of two inner cannulas that could be moved along outer guide cannulas by turning a small screw. Two bundles of 6 microelectrodes (32- and 64- $\mu \mathrm{m}$ diameter Formvar-insulated nichrome wires) were lowered through the inner cannulas to the coordinates AP -11.6 and -12.6, $\mathrm{LM} \pm 0.0$ and DV -0.2 of the Snider and Niemer (12) atlas of the cat brain and then glued to the top of these cannulas. Gross electrodes were implanted into the digastric, masseter and splenius muscles (the digastric and masseter muscles are the major jaw-opening and jawclosing muscles, respectively) and threaded into the parietal, frontal, temporal and retroorbital bones to record muscular activity (EMG), cortical activity (EEG) and eye movements (EOG). The microdrive and electrode implantation procedures are described in detail by Heym et al. (13).

Testing was initiated after a recovery period of 2 weeks following surgery. All microelectrodes were screened 2 to 3 times a day for the presence of stable, isolated singleunit activity (signal to noise ratio $>3$ ). In the absence of this activity the microelectrodes were advanced in small steps (about $80 \mu \mathrm{m}$ ) by moving the microdrive screw $1 / 4$ turn. When a recordable spike was found the animals were further tested. Neuronal activity as well as the EEG and 3 EMG were recorded during quiet waking, slow wave sleep and fast wave sleep, and while the subject was exhibiting spontaneous behaviors such as feeding and grooming. All signals were recorded on paper and on videotape. Spontaneous behaviors of the subjects and neuronal activity were recorded together on another videotape.

After advancing the microelectrodes about $5 \mathrm{~mm}$ into the brainstem, the animals were sacrificed and their hindbrain was processed histologically to locate the recorded neurons (for details see Ref. 13).

The data were treated as follows: 1) Neuronal action potential duration was measured. 2) Neuronal discharge was evaluated during $5 \mathrm{~min}$ of quiet waking, $5 \mathrm{~min}$ of slow wave sleep and $5 \mathrm{~min}$ of fast wave sleep. State-dependent changes in cell activity were determined by comparing firing rate across the wake-sleep cycle using the Friedman two-way test and Wilcoxon signed rank test. Regularity of cell discharge during quiet waking was determined by calculating the interspike interval coefficient of variation (standard deviation/mean). 3) Neuronal discharge and muscle activity were evaluated during $20 \mathrm{sec}$ of quiet waking, $60 \mathrm{sec}$ of drinking milk, $20 \mathrm{sec}$ of licking lips and 20 sec of licking forelimbs/washing face: a) A possible tight phasic coupling between muscle activity and neuron activity was examined by averaging full-wave rectified muscle activity $25 \mathrm{msec}$ before and $25 \mathrm{msec}$ after each spike across all the spikes, for drinking milk (this procedure is known as spike-triggered waveform averaging). The criterion for considering the existence of coupling was a post-spike muscle activity change with respect to mean pre-spike muscle activity exceeding by at least $50 \%$ the maximum pre-spike muscle activity change with respect to mean pre-spike muscle activity 
over a period of $1 \mathrm{msec}$. b) A possible gross tonic relationship between neuronal activity and muscular activity was determined by correlating the number of spikes per two 10sec epochs of quiet waking, drinking milk, licking lips and licking forelimbs/washing face with the corresponding integrated muscular voltages. c) A possible fine tonic relationship between neuronal activity and muscular activity was examined by correlating the number of spikes per six 10-sec epochs of drinking milk with the corresponding integrated muscular voltages. (For both b) and c) the Spearman rank correlation test was used.)

Seven neurons that showed a long-duration action potential $(>2.0 \mathrm{msec})$ and slow (2 to $7 \mathrm{~Hz}$ ) and very regular (interspike interval coefficient of variation <0.3) discharge during quiet waking were recorded in two animals. All of them systematically changed their activity across the wake-sleep cycle. The firing rate decreased from quiet waking to slow wave sleep (the respective means \pm SEM for six of these neurons were: $3.86 \pm$ 0.73 and $3.15 \pm 0.76 \mathrm{~Hz} ; \mathrm{P}=0.02$ ) and from slow wave sleep to fast wave sleep (the respective means \pm SEM for the same six neurons were: $3.15 \pm 0.76$ and $0.24 \pm 0.17$ $\mathrm{Hz} ; \mathrm{P}=0.02)$. One of these neurons was located in the dorsal third, 2 in the middle third and the remaining 4 in the ventral third of the medulla, in a stripe extending $0.5 \mathrm{~mm}$ to each side of the midline between the caudal and the rostral poles of the inferior olive. These landmarks delimit reasonably well the rostrocaudal extension of nucleus raphe pallidus and nucleus raphe obscurus in the cat (14). These neurons were classified as serotonergic on the basis of these functional characteristics and anatomical location (for criteria for identification of serotonergic neurons see Ref. 10).

Six neurons could be evaluated during a 60 -sec period of drinking milk. None of them exhibited phasic changes in its activity related to muscle activity.

All seven neurons were evaluated during quiet waking, drinking milk, licking lips and licking forelimbs/washing face. The discharge of these neurons tended to increase from quiet waking to licking lips to licking forelimbs/washing face and drinking milk. Muscle activity changed considerably from one behavior to another. Digastric muscle activity tended to increase from quiet waking to licking forelimbs/washing face to licking lips and drinking milk; masseter muscle activity tended to increase from quiet waking to licking lips to drinking milk and licking forelimbs/washing face; and splenius muscle activity tended to increase from quiet waking to drinking milk to licking lips and licking forelimbs/washing face. Five of the neurons changed their firing rate in relation to muscle activity. For 1 neuron the firing rate was related to digastric muscle activity $\left(r_{S}=0.77 ; P=0.04\right)$. For 2 other neurons the firing rate was related to masseter muscle activity $\left(r_{\mathrm{S}}=0.79\right.$ and $\left.\mathrm{r}_{\mathrm{S}}=0.79 ; \mathrm{P}=0.04\right)$. For the last 2 neurons the firing rate was related to digastric $\left(\mathrm{r}_{\mathrm{S}}=0.90\right.$ and $\mathrm{r}_{\mathrm{S}}=0.90 ; \mathrm{P}$ $=0.02)$ and masseter $\left(r_{S}=0.83\right.$ and $r_{S}=0.90$; $\mathrm{P}=0.03$ and $\mathrm{P}=0.02$, respectively) muscles activity (Figure 1).

Of the 6 neurons that could be evaluated during a 60 -sec period of drinking milk only 2 discharged in association with muscle activity. One fired in relation to digastric muscle activity $\left(r_{S}=0.90 ; P=0.04\right)$ and the other in relation to splenius muscle activity $\left(\mathrm{r}_{\mathrm{S}}=-0.88 ; \mathrm{P}=\right.$ 0.04) (Figure 2). The former neuron did not show any relationship between its activity and digastric muscle (or masseter muscle) activity when several behaviors were considered.

The main finding of the present study was the positive tonic relationship between the activity of presumed serotonergic neurons in the caudal raphe nuclei and the activity of masticatory muscles. This result suggests that at least part of the serotonergic influence on the trigeminal motoneurons is adjusted according to the required general intensity of contraction of the masticatory muscles during waking. 
Figure 1 - Gross relationship between the electrical activity of putative serotonergic neurons and the integrated electrical activity of two masticatory muscles (digastric muscle, top panel; masseter muscle, bottom panel). Two 10-sec samples were taken during each of the following behavioral activities: quiet waking, drinking milk, licking lips and licking forelimbs/washing face. $O$, neuron \#14041; $\triangle$, neuron \#24151; $\square$, neuron \#14021; 0 , neuron \#14173; $\nabla$, neuron \#14194. Correlations were significant at levels from 0.02 to 0.04

Figure 2 - Fine relationship between the electrical activity of putative serotonergic neurons and the integrated electrical activity of the digastric muscle (top panel) and the splenius muscle (bottom panel). Six 10-sec samples were taken during feeding behavior (drinking milk). $\diamond_{\text {, }}$ neuron \#14171; $\triangle$, neuron \#24151. In both cases the correlation was significant at the 0.04 level.
Serotonin by itself does not activate lumbar (15) and facial (16) motoneurons but renders them more responsive to glutamatergic excitation. Katakura and Chandler (6) reported the same facilitatory action of serotonin on digastric motoneurons. According to Vandermaelen and Aghajanian (16), serotonin may cause membrane depolarization
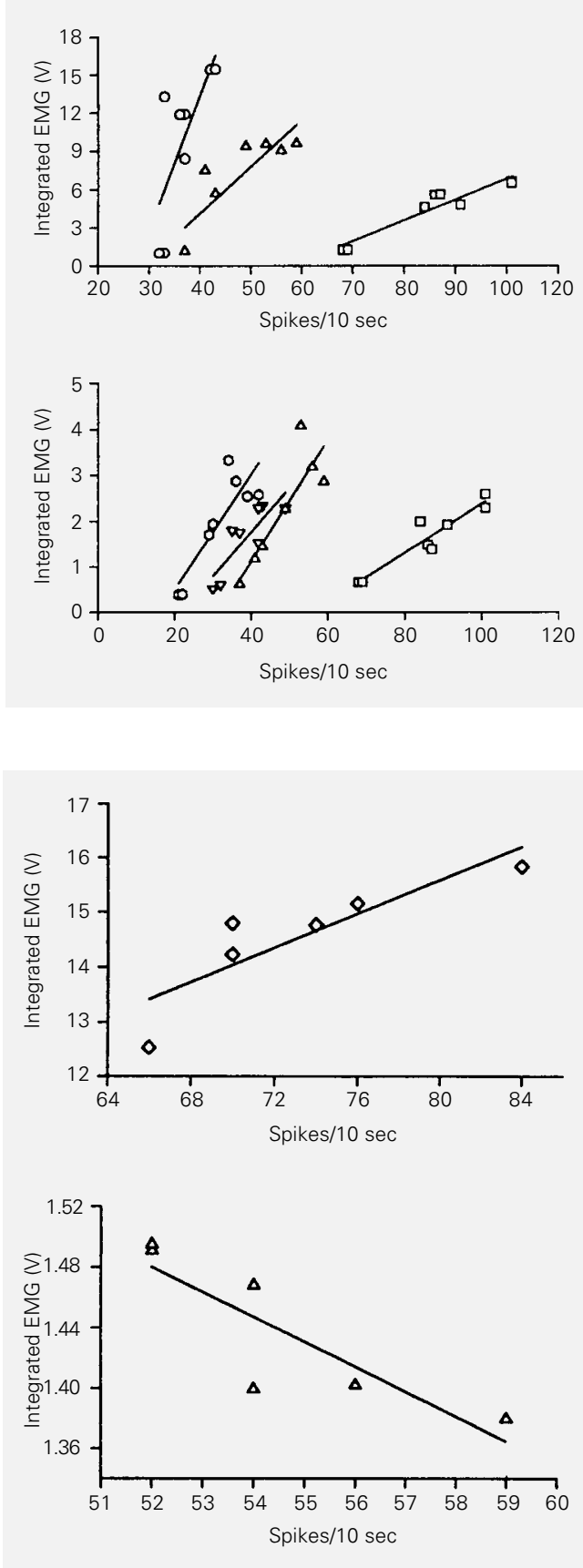

by decreasing resting membrane conductance to potassium ions. We propose that the serotonergic input, possibly by modulating the depolarization level of the trigeminal motoneurons in a tonic and graded way, actively contributes to the determination of the general intensity of masticatory muscles contraction appropriate for each behavior.

We observed only one case in six of fine tonic coupling between neuronal firing and masticatory muscles activity but five cases in seven of gross tonic coupling. This observation suggests that the presumed active serotonergic modulation of trigeminal motoneurons excitability may be more important for causing coarse general changes in muscular activity than for causing fine ones. It is possible that serotonin specifically facilitates the recruitment of additional, higher threshold, trigeminal motoneurons by the central pattern generators that control jaw movements.

Also highly significant was the fact that in a sample as small as 7 neurons we could identify 6 neurons whose activity was related to jaw muscles activity. This fact correlates well with the anatomical observation of a very high density of serotonergic projections from nucleus raphe pallidus and nucleus raphe obscurus to the trigeminal motor nucleus $(1,2)$. Veasey et al. (11), also using cats, recorded 29 serotonergic neurons in nucleus raphe pallidus and nucleus raphe obscurus. Twelve of these neurons increased their discharge during feeding (licking pureed meat and water). The much smaller percentage of serotonergic cells presumably related to the control of jaw movements found in their study could be explained by the fact that the subjects exhibited only one behavior with these movements and probably developed the same general intensity of masticatory muscle contraction.

It is not known whether the serotonergic neurons that supposedly influence masticatory muscles activity also participate in the control of other body muscles. Since five of 
the neurons related to the masticatory muscles were not related to the splenius muscle, some degree of specificity is suggested. Only one of 10 feeding-responsive serotonergic cells tested by Veasey et al. (11) was not activated during a treadmill-induced locomotion task. However, their finding is not conclusive because during locomotion jaw position must be actively held against destabilizing inertial and gravitational forces. Serotonergic activity may have been increased by higher nervous centers to augment the gain of the stretch reflex of the jaw-closing muscles and, consequently, stiffness of muscles (17).

No change in jaw muscles activity phasically related to serotonergic neurons activity was found in the present study. This is not surprising since the action of serotonin on motoneurons has been described to be of slow onset and to have a prolonged time course $(6,15)$. Related phasic changes in serotonergic neurons activity and physiological functions were observed only rarely in other studies $(11,13)$.

The results of the present study are in agreement with the views of Jacobs and Azmitia (8) that the serotonergic system has a tonic modulatory role in the nervous system. Jacobs and Fornal (18) proposed that one of the primary functions of the serotonergic system is to facilitate motor output. Our main finding supports this hypothesis.

\section{Acknowledgments}

We thank Mr. Roberto Vieira and Mr. Ademar Petri Filho for their valuable technical assistance.

\section{References}

1. Steinbusch HWM (1981). Distribution of serotonin-immunoreactivity in the central nervous system of the rat - cell bodies and terminals. Neuroscience, 6: 557-618.

2. Cropper EC, Eisenman JS \& Azmitia EC (1984). 5-HT-immunoreactive fibers in the trigeminal nuclear complex of the rat. Experimental Brain Research, 55: 515-522.

3. Fritschy J-M, Lyons WE, Molliver ME \& Grzanna R (1988). Neurotoxic effects of $p$ chloroamphetamine on the serotoninergic innervation of the trigeminal motor nucleus: retrograde transport study. Brain Research, 473: 261-270.

4. Fort P, Luppi P-H, Sakai K, Salvert D \& Jouvet M (1990). Nuclei of origin of monoaminergic, peptidergic, and cholinergic afferents to the cat trigeminal motor nucleus: a double-labeling study with cholera-toxin as a retrograde tracer. Journal of Comparative Neurology, 301: 262-275.

5. Kolta A, Dubuc R \& Lund JP (1993). An immunocytochemical and autoradiographic investigation of the serotoninergic innervation of trigeminal mesencephalic and motor nuclei in the rabbit. Neuroscience, 53: 1113-1126.
6. Katakura N \& Chandler SH (1990). An iontophoretic analysis of the pharmacological mechanisms responsible for trigeminal motoneuronal discharge during masticatory like activity in the guinea pig. Journal of Neurophysiology, 63: 356-369.

7. Ribeiro-do-Valle LE, Metzler CW \& Jacobs BL (1991). Facilitation of masseter EMG and masseteric (jaw-closure) reflex by serotonin in behaving cats. Brain Research, 550: 197-204.

8. Jacobs BL \& Azmitia EC (1992). Structure and function of the brain serotonin system. Physiological Reviews, 72: 165-229.

9. Ribeiro-do-Valle LE, Fornal CA, Litto WJ \& Jacobs BL (1989). Serotonergic dorsal raphe unit activity related to feeding/ grooming behaviors in cats. Society for Neuroscience Abstracts, 15: 1283.

10. Fornal CA, Metzler CW, Marrosu F, Ribeiro-do-Valle LE \& Jacobs BL (1996). A subgroup of dorsal raphe serotonergic neurons in the cat are strongly activated during oral-buccal movements. Brain Research, 716: 123-133.

11. Veasey SC, Fornal CA, Metzler CW \& Jacobs BL (1995). Response of serotonergic caudal raphe neurons in relation to specific motor activities in freely moving cats. Journal of Neuroscience, 15: 53465360 .
12. Snider RS \& Niemer WT (1970). A Stereo taxic Atlas of the Cat Brain. University of Chicago, Chicago.

13. Heym J, Steinfels GF \& Jacobs BL (1982). Activity of serotonin-containing neurons in the nucleus raphe pallidus of freely moving cats. Brain Research, 251: 259276

14. Jacobs BL, Gannon PJ \& Azmitia EC (1984). Atlas of serotonergic cell bodies in the cat brainstem: an immunocytochemical analysis. Brain Research Bulletin, 13: 1-31.

15. White SR \& Neuman RS (1980). Facilitation of spinal motoneurone excitability by 5-hydroxytryptamine and noradrenaline. Brain Research, 188: 119-127.

16. Vandermaelen CP \& Aghajanian GK (1982). Serotonin-induced depolarization of rat facial motoneurons in vivo: comparison with amino acid transmitters. Brain Research, 239: 139-152.

17. Lund JP, Drew T \& Rossignol S (1984). A study of jaw reflexes of the awake cat during mastication and locomotion. Brain Behavior and Evolution, 25: 146-156.

18. Jacobs BL \& Fornal CA (1993). 5-HT and motor control: a hypothesis. Trends in Neuroscience, 16: 346-352. 